\title{
Visibility as a key concept in Communication and Media Studies
}

\author{
Samuel Mateus \\ Madeira University / Labcom.IFP; CIC.Digital \\ E-mail: samuelmateus@uma.pt
}

\begin{abstract}
The concept of visibility has become a problematic one as hypervisibility gave rise to new forms of opacity that are formed not through secrecy but by its opposite, pan-visibility. Paradoxically, by amplifying visibility, media create new forms of invisibility. An analysis of visibility will provide us with a precise perspective how these processes occur. In this paper, we suggest three lines of empirical and theoretical investigation in the topic of visibility: a sociological (symbolic) axis; a collective (publicness) axis; and a technological (media) axis. Since the social category of visibility is a central aspect of communication and media studies, we will be interrogating it through three distinct ways: visibility as

constitution of different regimes of visibility; visibility as a pivot-concept of publicness since it is this public quality that transforms proto-visibility into a full accomplished visibility; and, third, the transmutations and dangers stemmed from media's production of visibility. Each one of these principles highlights different concepts: in the field of visibility we need to address inter-visibilities; in public visibilities we need to address proto-visibilities in verge of becoming full-visibilities through the synchrony of collective attention; and in mediated visibility it is imperative to deal with super-visibility as an extreme effect of an intense modulation perpetrated by communication technologies.
\end{abstract} a field whose symbolic determination results in the

Keywords: visibility; visual; publicness; media.

Even in the case of things which are clearly visible, you know that if you do not turn your mind to them, it is as though they had never been there or were far away.

(Lucretius, On the Nature of Things, IV, 809 apud Van Winkel, 2005: 7)

\section{Introduction}

T $\mathrm{N}$ one of his essays, Walter Benjamin (1999: 734) described the glass as the main enemy of 1 mystery and a fierce opponent to unique distance. The transparency, hardness and sobriety of glass allows no traces, no secrets, and all becomes visible. It became the preferred material to build

A Revista Estudos em Comunicação é financiada por Fundos FEDER através do Programa Operacional Factores de Competitividade - COMPETE e por Fundos Nacionais através da FCT - Fundação para a Ciência e a Tecnologia no âmbito do projeto Comunicação, Filosofia e Humanidades (LabCom.IFP) UID/CCI/00661/2013.

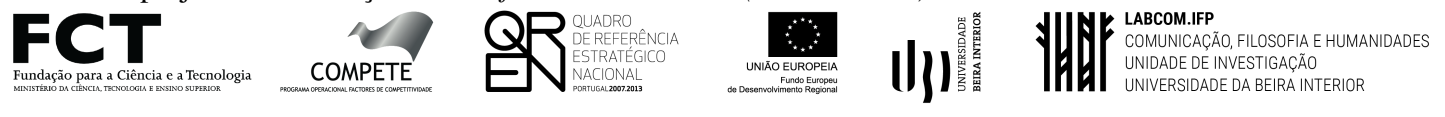


the modern world as we see it in building's façades. Indeed, our culture features the apology of the visual where everything becomes transparent (Sennett, 1992): our relationships, our buildings, our workspaces, the annual income of politicians, even privacy seems to disappear in the public exposure of reality- television.

No generation before has been so fascinated by visibility as ours and media technologies do confirm that: photography may be taken as an attempt to render visible the physical reality; cinema may be perceived as an effort to render visible the imaginary; and television is possibly a medium trying to render visible ordinary life. Our mediatized societies are accustomed to believe in its eyes.

In a certain sense, contemporary societies embody a giant and pervasive mythological figure: Argos, the giant whose epithet, "Panoptes", "all-seeing", labeled his numerous eyes. According to Ovid, to honor her faithful watchman, Hera had the hundred eyes of Argus well-looked-after in a peacock's tail. We are still the heirs of Argos in respect to total, all-mighty visibility. CCTV images are perhaps one of the most discussed realizations of the all-seeing eyes whose major asset is permanent scrutiny. Today, media surprisingly provide us with the hundred- eyes of Argos from computer screens through television to social media. In some respects, it seems we are in the verge of losing the anthropological structure of invisibility. Debray (1992), for instance, is one of the few to alert us to the emancipation of the visible and the consequent extinction of the invisible. Visibility is today such a value that we tend to related with sincerity (the human character becomes notorious), authenticity (emotions becomes notorious), immediacy (the disappearance of the medium becomes conspicuous) and transparency (the arcanum imperii is constantly exposed).

We are in the middle of a social dilemma where excess leads to annihilation just as too much light impairs us from seeing things accurately. In the same way, as disproportionate noise negatively impacts communication, hypervisibility may mask reality. The excess of visibility - one may called it a superlative or an extra degree in visualization (Van Winkel, 2005: 15) - envisages an important paradox: by render certain things visible, it tends to obliterate others. Hypervisibility supposes blindness in its own conditions of functioning. Blanchot or even Lacan have both remarked how an encompassing visibility supposes a blind spot to the access of the present and the full experience. Every time we see all, we do not see everything because there is an accursed share or an excess (Bataille, 1988) surmounting the hegemonic gaze. Every time we publicize privacy there is a rest that is not invisible but beyond visibility.

Media have amplified this paradox and present the world as if the medium could be erased: they create a strong familiarity feeling while obliterating their own mediation. Adorno (1954), for instance, regarded television as a social instrument of opacity while appearing to function with total immediacy. On the same token, Innerarity (2004: 53) contends that the transparency of modern media produce a specific blindness as the profusion of images and words saturate reality with an undifferentiated mass of facts. When we see everything, reality becomes thick but disorienting. When things are disproportionately seen, we cannot see beyond what is presented to us. Behind the appearances there is an enormous unseen space. This is the paradox Innerarity (2004: 54) points to: hypervisibility gives rise to new forms of opacity that are formed not through secrecy but by its opposite, pan-visibility. Today, to conceal does not mean to hide. Concealment 
may simply be in its mode of rendering the world visible. We may block out something precisely by an excess of showing.

By privileging total transparency and by making the world a glassy reality (to quote Benjamin), it is no more secrecy, the hidden, the underground or the clandestine that is presented to us in a problematic way. Visibility becomes a problematic notion (Innerarity, 2004: 52) not because concealment threatens it, but mostly because visibility has been transformed in a kind of ideology of transparency that dissolves the conventional frontiers between visibility and invisibility. To see is to problematize. In other words, visibility becomes a problem in the moment it is object of a strategic use and management.

In his novel Blindness José Saramago almost deals with this paradox by alluding to a pure visibility: "Perhaps only in a world of the blind will things be what they truly are". Today we are dealing not with the end of invisibility but mostly with the end of the traditional opposition between secrecy and the manifest, the hidden and the exposed. Visibility does not simply contrasts with invisibility. The visible and the invisible form a dual expression in the management of visibility. Emergent visibility is the result of the incessant intersection between visible and invisible forces, the end product of different regimes taking shape in unpredictable manners. Visibility regimes (cf. Mateus, 2014) affords new collective dynamics requiring new ways to think its influence in today's society.

By referring to visibility as a problematic notion we are also stating a conundrum of visibility (Boyd \& Marwick, 2009). In other words, the concept of visibility contains new complications as media heighten the potential for visibility. Yet, by amplifying visibility they create new forms of invisibility. An analysis of visibility will provide us with a precise perspective how these processes occur. But it will not offer us an understanding of the key importance of visibility to media studies.

In this paper, we take on an assessment of the conundrum of visibility regarding its problematic relationship with media and communication technologies. We will advocate that not only visibility is a main concept to communication and media studies, as also it condenses a fundamental category to social sciences. By acknowledging this double feature, we are better equipped to describe many social questions (surveillance, recognition, social theory, etc). But, above all, we acquire a renewed perspective on the idea of publicness one that should be strongly connected with media and visibility.

Thus, we will argue media is an inescapable dimension of today's visibility and that one of the main advantages to consider visibility in relation to publicness is related with the possibility to envisage a collective synchrony of attention. We need to consider visibility within a social theory of communication media. We will put forward three axes through which a critique of communication and media may emerge: visibility as a modality of representation; visibility as a pivot condition of publicness; and mediatic visibility as one of the most pervasive and riskier kind of social visibility. These are three motives that mark the important of such a notion to media studies and that justify its study in today's societies.

To that effect, we will start by address the field of visibility (section one), define its associations to publicness (section two) as well as to comment upon media visibility and its effects (section three).

Estudos em Comunicação, $\mathrm{n}^{\circ}$ 25, vol. 2, dezembro, 2017 


\section{Visibility as a Field}

Although visibility is today an omnipresent concept there are still distinct meanings being confused. And it is the usual indistinction between a literal and a metaphorical meaning that has limited the use of visibility in media studies. In fact, some authors such as Thompson (2005) tend to regard visibility in terms of the immediate sensory sphere and that's why he proposes to call a"new visibility" to the process that makes this sensory experience of visibility as non-presence, mediated sensibility.

Still, visibility has today not just a literal meaning as immediate sensory experience but also a metaphorical one where the concept assumes a set of symbolic meanings normally related to the way particular events are communicated by media. It is in this sense that we can relate social recognition to visibility (Brighenti, 2007: 329) and explore the ways social agents demand media attention to become socially acknowledged. It is also at this light that one may understand the fierce labor to come a celebrity (from singing and acting until reality television tasks such as discovering the "secrets" of other competitors). Celebrity is, thus, described as a personality whose visibility concedes him an aura of admiration and approval.

In order to better apprehend the literal and metaphorical meanings of visibility in contemporary societies, we want to advance the idea that the difference between these two layers of visibility is not a difference of nature but a difference of degree.

In other words, visibility as a sensory experience and visibility as a symbolic experience do not exclude each other. They are not expressions of visibility's polysemy. Instead, we agreed with Brighenti (2008: 4) when he suggests to deal with visibility as a complex phenomenon alternating different modes of seeing. This means that the sensory dimension and the symbolic dimension of visibility intermix together implying separate modes of seeing the world. Visibility happens according to this dual disclosure and its fundamental ambiguity comes precisely from the permanent interweaving of its sensory and symbolic components. Visibility is not simply an appearance or an image; visibility is also a social process in itself engaging their social commitments, each one with their own jurisdiction.

It is also because of this constitutive ambiguity that media are key objects to visibility. Media constantly mix the sensory experience of visibility (making something visible and perceptible) with its symbolic dimension (making something collectively recognizable and notorious). Social media constitute a special problem since they are one of the main forms this interweaving occurs now. Sometimes there are not much to recognize in what social media show (ex: humorous memes). Other times there are not much to display and to make visible (ex: personal live pictures as me eating pizza at home). Yet, we easily tend to give to what is merely visible our approval (ex: sharing memes in Facebook), as well as we tend to acknowledge what is not necessarily recognizable (ex: "Like" in Facebook to the picture of me eating a slice of pizza).

This is also a main theme on celebrity studies: many so called stars gain their social approval because media tend to function on this dual dimension of visibility. So, some personalities are visible in traditional and new media and become an object of reverence (ex: the socialite Kardashians) followed by millions. And they are recognized as important personalities (ex: being invited to attend some elitist events) because they are always keeping appearing in the social imaginary. 
The sensory dimension of visibility feds its symbolic one and, in return, its symbolic dimensions reinforces and justifies its sensory visibility.

So, why not, like Innerarity (2004: 130) or Brighenty (2010), to think contemporary societies from visibilities relations? Why not to critical ponder on social relationships through the very notion of visibility? What could we understand if we would include "visibility" to the pantheon of social theory ${ }^{1}$, and specially to the vocabulary of the social theory of communication and media?

In order to lead off this approach, it is useful to place visibility as an ecology or a field of separate and complex actions. Visibility is not just a social category to apprehend human interactions, it may also be seen as a special arrangement of visible and invisible movements encompassing disclosure and cloaking operations through which one moves in social interplay. Visibility is a field that helps to shape subject positions and as such contains a strategic nuance. To make society visible to itself is a crucial operation to social theory.

Visibility may, thus, be considered as a field highly interdependent of complex social, technical and political arrangements (Brighenti, 2010: 3). Communication technologies certainly play a part in how the visible is inscribed in social reality, and how visibility suffers different modulations pointing to its double-swords aspect: it has both an (potential) empowering as well as a (potential) disempowering role. It may suffice to say that visibility may impel social movements to social acceptance (ex: American Civil Rights Movement), as also visibility may contribute to the panoptic control CCTV images impose on individuals.

Visibility may, then, be best described as having a rippling effect on society and part of this effect comes (not exclusively) from the ways media set up empirical visibilities and influence visibility's contingent compositions, recomposition and inter-compositions. In other words, "the field of visibility is relational, strategic and processual" (Brighenti, 2010: 39).

The field of visibility supposes a process of inter-visibility, that is, a positional (ergo, strategic) quality working upon thresholds of collective attention whose final result promotes a means to sorting, ordering and classifying of events and individuals. Visibility as a social field designates how visible relationships structure and attribute intrinsically relational and ambivalent social positions to individuals and their actions. This is to say visibility concerns the management of collective attention by putting into circulation different articulations of the visible.

To mention how visibility may be displaced and articulated in an interdependent field take us to envisage an ecology of reciprocal visibilities organized into regimes of visibility (Thibaut, 2001; Mateus, 2014). Brighenti for instance, offer us a strict definition of those regimes of visibility: "a regime is a repeated, agreed upon and more or less settled pattern of interaction" (Brighenti, 2008: 25). Each visibility regime would be appreciated by the conditions it puts forwards, namely, what is worth seeing, what we should see, what others must see or what is right for us to see. Visibility regimes articulate their own "visibility games" where certain positions are permitted (ex: in reality shows like Adam looking for Eve or Big-Brother spectators are allowed and even encouraged to peep) and others ferociously prohibited (ex: it is not consecrated by law, at least in some European countries, that the face of children be filmed in cases about the legal dispute of parents or when

1. Brighenti (2007: 324) argues that "visibility can be counted as a fully entitled sociological category". 
they are victims. In those cases, media cover up their faces even if they still record images of them).

To the sum of the interdependent and ambivalent effects of each visibility regime we call a field of visibility where tensions and disputes over the visible decisively contribute to establish its normative conditions. Visibility is an enabling resource whose division between sensorial and symbolic layers need to be apprehended together. Talking about a field of visibility as the purpose to designate the interplay of thresholds of visibility capable of displace social positions and be used as a strategically resource for selectivity, regulations and stratification of individuals and events.

A field of visibility encloses a perspective on the concept where he can be viewed as a modality of social representation. By moving through different positions, visibilities are never a fixed phenomenon but a highly dynamic and constructed process being use by individuals to assert and represent themselves. There may be panoptic and synoptic asymmetries between the majority who see and the majority who is being seen (Mathiesen, 1997) or may possibly be distortions of visibility when a person is exclusively visible in a given social situation. This only adds to the understanding how visibility is such a disputed category ${ }^{2}$ in constant construction (Champagne, 1993).

Very simply stated, shaping and managing visibilities is a huge work that we are used to endeavor. By naming a social field made by different regimes of articulation of visibility we acknowledge that visibility is not just a question that rose with modern media. It is more than a technical matter; it is also a social and political matter that puts perceptivity along with visibility as two primordial notions.

As long as we talk about reciprocal visibilities and field of visibility we are also addressing the question of perceptivity. To address it is to refer to a new kind of definition of the visible. By defining visibility as an ecological system or a field we are positing that the visible is something more than simple visual. This definition we are talking about here concerns the dual nature of visibility taken as sensory and symbolic experience. Just as visibility is not just rendering something present to the optic senses, the visible is also not just something visual. The same way visibility contains a symbolic dimension, we will use Brighenti's strong definition and talk about the visible "is the prolongation of the visual impregnated with the symbolic" (Brighenti, 2010: 32). This has tremendous consequences.

First of all, it means, visibility is not something static or pre-determined by the individual's or event's self-properties. Visibility is not about showing something there but mostly about making something showable. In other words, visibility needs to be worked upon in order to become possible to visualize it. Visuality need to be become visibilised. In order to collectively exist objects need to become visible. The visible is something composed, constructed and performed. It points to the symbolic construction of the visibility. To show a person in a photograph (ex: Cristiano Ronaldo photographed in Paris Disneyland by paparazzi) is not to capture visibility: it is to render it subject to a symbolic construction of the visible (including how is it technologically captured and framed; but also socially reproduced, published, and reported). Symbols, including visual

2. Not only in modern times but we can also trace the centrality of visibility to Ancient Times where coinage in Rome were used as a form of taken abroad to the Roman Empire the image of the Emperor. 
signs and other semiotic categories renders things visible. We should ask not just how images gestures, objects and representations symbolize collective identity, social cohesion, shared values, etc., but also how we use visibility to make symbols special operators of social relationships (cf. Brighenti, 2010: 32).

Second, when we picture the visible as the prolongation of the visual impregnated with the symbolic, we are emphasizing the field of visibility, namely the dual nature of visibility and the hybridity present in the possible catenations and articulations of the visible. Concurring in the previous point, we will say that there is no pure visibility or pure invisibility. By stating a field of visibility one is accepting that visibilities are social constructions in which the symbolic takes part.

The proof to this impossible pure visibility, one without a symbolic impregnation of the visual, is that visibility is a problematic notion since the first human societies. And the need to include media in the understanding of today's visibility only confirms that the visible is not the visual; visibility has its richness as a social category in its contaminated form with the symbolic and the strategic use of the visible to empower or disempower individuals and institutions.

So, the first reason we advance to put visibility within the scope of communication studies is that visibility is not a question about showing something visual but making something showable. And this making of showiness, this rendering the visual visible is not without a symbolic dimension.

We end this section returning to the beginning: visibility is not just to display a thing and make it a sensory experience; visibility is to render it visible through diverse regimes of reciprocal and interdependent gazes. It is to look with intention. It is to construct perceptions and this means we must acknowledge it as a core notion in the social theory of communication.

\section{Visibility and Publicness}

We have just argued the problem of a public visibility is concomitant to the idea of visibility being a field through which something is built to be displayed and becomes showable. To make the visual entering the ecology of visibility is necessary to think the concept in its symbolic dimension. One key area of such symbolization of visibility lies in the public realm.

Public realm means here, not a public sphere or an ethical and normative publicity (Habermas, 1991). We know how political philosophy have traditionally referred public sphere and visibility (Weintraub, 1997) from a deliberative and proceduralist standpoint on democracy. We also recognize how publics fight to become visible not just to the State but also visible to themselves, open to society and its problems (Dewey, 1927).

Studying visibility involves to consider how it depends on arenas of inter-visibility and social interaction, that is to say, we need to consider visibility and publicness interpenetration. Thus, a core aspect of visibility to media and communication studies lies in its deep relationship with publicness, a notion without which it would be impossible to suppose reciprocal visibilities in society.

Publicness is the simply quality of being public, open, accessible to a collective scrutiny. It is the sentiment of the collective, of the plural definition of individuals - to allude to Figurational 
Sociology (Elias, 1978). Publicness may be defined through a property Tarde (1901) has sharply noted on the age of the publics: the mutual impact or reciprocal influence of distinct minds converging into action at spatial and temporal distance. In other words, publicness stands for a social quality enabling acts of reciprocation in territorial dispersion which produces synchronic configurations of social interactions.

Publicness is a central notion to the category of visibility because it represents an aesthetic and symbolic synchrony of attention. Visibility and Publicness go hand on hand because they operate together the arrangement of inter-visibilities. Since the visible is a symbolic visuality, we need to consider publicness as the quality capable of providing a symbolic realm where visibility is constructed in a synchronic way. When a happening becomes public it has not just passed the frontier that makes it visible to some, it has also surpassed a threshold of synchronic attention. In this sense, visibility entails a public aspect and that's why it is an important object to communication studies.

Like visibility offers an interdependency of gazes, publicness registers a relational form of those gazes. Publicness turns visibility a social dimension. By producing different forms of synchrony of attention (either mediatized and non-mediatized) publicness catalyzes the explosion and the implosion of related visibilities. Inter-visibilities are ordered and configured due to the possibility of synchronizing perceptions and prolongate collective attention into frames composing the regimes of visibility.

At this regard, publicness is a realm of communication through mediatized but also nonmediatized visibilities. And, according to what has been said, visibility is a field of coordination of attentions that needs publicness to be collectively configured. It is with the idea of publicness that visibility does attain the accessibility necessary to become an arrangement of inter and reciprocal visibilities. It is also a realm of shared observation, of communed views whose porosity renders to the perceptive activity a central instrument to social partaking and collective sharing. Society is institutionalized as multiple publicness realms. Are those realms that, together, subsidize the forms through which visibilities become acknowledged, understood and shared in joint expressions of social activity. Thus, the hypertrophy or atrophy of inter-visibilities is intrinsically related to publicness.

As an example, let's consider the visibility of a pop-star singer. Without some sort of publicness, that is, synchrony of attention, its own visibility would simply be invisible. In order to become a visible element on a galaxy of other well- talented elements, the pop singer need to gain attention. He only gathers visibility if he recollects the dispersed attentions of individuals. So, he needs to become a public figure, that is, a personally able to synchronize the collective gaze in public configurations that accommodate its pre-existent visibility. It is no surprise that You Tube transmuted anonymous persons into music stars: indeed, what the social media has done was to give sheer and literal mediatized visibility (video images) a symbolic and public visibility (social notoriety). Without its massive appeal (of symbolizing images), and without its capacity to synchronize attention (publicizing images), You Tube would not matter. He would just emulate or put into circulation a proto-visibility. It would transmit videos of persons singing but it is just when You Tube gives notoriety and offers millions of visualizations to those videos, that You Tube transforms proto-visibility into a full accomplished social visibility. In other words, a full accom- 
plished visibility needs to be symbolized (ex: me singing in order to become a pop idol) but also needs to be publicized (ex: me singing being seen by millions in order to become a pop idol). We have seen without symbolization visibility does not override the visual. But without publicness visibility does not achieve collective status. By synchronizing collective attention, publicness enables visibilities to transform into inter-visibilities, into share notions of what is not just able to be seen, but also able to do itself capable of putting others seeing.

Put in other way, publicness constitutes a symbolic ecology inhabited by both the particular and the plural, the individual and society. That's why we need to consider the notion of public. The public is the constant appropriation of social experience (Mateus, 2011). Publicness is not necessarily harmonious or ideal (publicity) neither hegemonic (a public sphere). Consequently, the public is always a deep collective experience encompassing symbolic gazes but also factic communication and haptic forces. It is a collective force capable of shaping social bodies of knowledge, affects, conflicts or appeasements. It is pulsation, a pacing non-individual, non-collective entity.

Away with a capital, unitary, self-imposing Public of normative theory of publicity, visibility pushes us towards a lower-case, emotional-charged public. A public seen as a more spontaneous happening, occurring whenever social experience is at stake. We cannot be the public. Simply recall how historical approaches to the public sphere put it as idealized (Kramer, 1992). Yet, we can be in public. A public exists when feeling and belonging converge into registers of interaction, be it mediatized or not. And one major asset of publics is that entails regimes of visibility that decompose their appearances and that are related, for instance, to the emergence of notions such as counter-publics (Fraser, 1992), bourgeois public (Habermas, 1991) or proletarian public sphere (Negt \& Kluge, 1993).

But what authorizes us to relate visibility, publicness and publics is that they all suppose some kind of commonality. It is social partake and shared belonging that wraps up them all. Bridging and bonding are common denominators in these concepts.

From a visibility standpoint, the public may be defined as the object in which synchronicity of attention (Brighenti, 2010: 117) characteristic of publicness takes place. From a visibility perspective, the public is appropriately conceived by Brighenti (2010: 74) as "a spatially dispersed but attentionally and emotionally synchronized social territory". It is best described as a symbolic aesthetic incidence, not a determined group of persons, nor a distant crowd: in publicness, the public runs through society reclaiming different visibilities. It is in publicness that visibility and the public emerges as central analytical tools since publicness means openness and accessibility (ex: the public eye). By entering publicness, one becomes a subject of visibility; someone who is turning visible into others has also becoming a central piece of interaction. And it is because the boundaries of publicness are constantly shifting that we can refer to the visibility regimes and its unboundedness. Words but also gazes resonate in publicness. Rationality but also visibility constitute publicness taken - as Brighenti (2010: 125) remarks - as a relational field of attention and affections. Just like visibility is some sort of aesthetical contagion, publicness is a field of contagious ideas and its dissemination. Without visibility, political subjects are not drawn. Without publicness, there are not subjects to look and aknowledge upon. 
So, until now we have stated visibility supposes the symbolization of the visual and that full visibility is only attainable within a public process. But how does one should envisage public visibility today? The answer is fast and inescapable: media.

In fact, one of the most significant types of visibility is the media-type whose ubiquity and intense rhythm of functions (cf. Virilio, 1986) induces fundamental alterations to visibility regimes. Of course, the relation between media and visibility are not one-sided. On the contrary, they are complex - promoting liberating relations of visibility but also oppressive relations to them.

In the next section, we confer how the potential to visibility brought by modern audiovisual media influences visibility and its publicness. By integrating the wide effects of media in visibility we disclose a third reason to consider it in communication and media studies.

\section{Mediated Visibility}

One of the main effects of media in the idea of publicness was that they opened up public scrutiny and expanded publicness in tremendous ways. The erosion of the public and private Habermas (1991) pointed out suffered a suddenly impulse. "Thanks to the media, these previously hidden practices and events had been given an entirely new status as public" (Thompson, 2005: 31). With media, we witness the explosion of visibilities and a whole new world of mediated visibility has come to stay.

Mediated visibility is not just a leaking form of the visible; it is also an escalation of the visible realm with an increasingly difficulty to govern it. Visibility ceases to be locally situated (those who see and those seen share the same spatial location) as well as temporally situated (those who see may see outside the temporal framework of those who are seen) (Thompson, 2005: 35). With the development of communication media, visibility frees itself from the physical and temporal circumstances. We witness events occurring in distant places in real time, live time, but we can also witness events occurred in the past and that can be re-presented in the present time. This points to the preservative quality of media and show how media should be thought in terms of mnemonic practices.

At the same time, the sense of sight loses its physicality and becomes more an abstract entity. While in face-to-face interactions, sight is the operational instrument to see, in mediated communication sight is just one of the operational instruments along with a visual technology (ex: computer, television, smartphone, cinema screens, etc).

Since it involves the participation of technical devices, mediated visibility is tendentially unidirectional. Even in the case of tele-conferences or skype sessions, individuals may only see what the camera records. There are two images that juxtapose but that do not overlap. One can even be seen without see. This clearly happens when distant others are filmed or photographed or caught on surveillance cameras. We are talking about a "de-spatialized simultaneity" (Thompson, 2005: 37): distant others are rendered visible in the same time-frame and can be seen at the exact moment they appear. But we must also include a "mediatized co-presence" (or mediated quasi-interaction (Thompson, 2005: 37)) where others that may have been dead for decades appear on our eyes.

The rise of television, first, and internet, then, has amplified the social significance of these new forms of visibility created by media. Those forms may be described as dissemination and 
irradiation processes that expanded the importance of visibility vertically (in terms of quantity) but also horizontally (in terms of a qualitative perception). "Mass-media are high-visibility places endowed with the quality of conferring visibility to the people who join them. As a process, visibility works instantly but extends in time (...). We may say that visibility has a flash and a halo: it is both instant and it has a duration" (Brighenti, 2007: 332). So, relating to these terms, visibility is vertically important when it conceded a flash of collective attention to the visible object (today's 15 minutes celebrity) but also horizontally relevant at the moment that the visible is a prolonged and lasting visibility. Think on Marilyn Monroe or Elvis. They are no more vertically visible (they have died so we cannot see them in face-to-face interactions) but they have a persisting allure that made them entered this horizontally or hallo visibility that enables us to constantly see them over and over. They have been subjects of a collective memorialization in which mediated visibility have a key role.

So, today's visibility in a mediatic era has both this intense, spontaneous and prompt side (a flash ou vertical visibility); but simultaneously a slow, more stable and longstanding side (a hallo or horizontal visibility). Advertising would be an example of a high-intensity, vertical visibility while pop idols such as Madonna would exemplify this long-term, enduring visibility.

Mediated visibility is the consecration of new forms of management of visibilities in a time where a visibility of co-presence has become secondary. Most important, these new technological forms actively contribute to visibility regimes as they make something showable through the symbolization of the visual. Just like seeing is always shaped by the broader cultural frameworks, media are key players in setting up those socio-cultural assumptions. Then, media may possibly be taken as an update to the field of visibility that foster an expansion and a reformulation of visibility. In other words, the possibility to repeat presentification of the visible over time (influencing collective memory) tremendously expands the field of visibility.

Visibility, publicness and media are inder-dependent. Because publicness proceeds to the synchronization of attention necessary to attain a full visibility, one should not forget the role media have in the modulation of that public synchrony of attention. Media are today the chief distributors of visibility relationships (Innerarity, 2004: 142).

Contemporary media produce two chief modulations of attention: the primacy of active attention and the primacy of passive attention.

Until now, traditional visibility arrangements by media like television or video worked mostly according to active attention: the possibility of seeing everyone without being seen. Visibility is active since it makes something seeable yet it does not show who is seeing (just think in the appeal to see your favorite actor in his new movie, for example). This is also the case of television or even surveillance footage. We can see them all but they cannot see back. The focus is in the ability to see and in the making of an imposed visibility. This is somewhat related with the inversely proportional quantity and time: as perception possibilities increase, it decreases the possibilities of knowing that we are being potentially watched ${ }^{3}$.

3. This is better illustrated, for example, by computer cameras which can be switched on remotely by hackers and record the room where the camera is located. 
This asymmetric visibility implies the possibility of social control and may stand as one of the most important instruments on political resistance (Brighenti, 2010: 185). But by talking about the active modulation of attention we are emphasizing the primacy of visibility as a capturing process who can select, highlight and change the perceptions of reality.

In contrast, today mediated visibility acts likewise according to $a$ passive attention where the focus is not in the capacity to totally see but in the possibility of being totally seen. Beyond visibility as control, there is a subtler modulation of visibility perpetrated by media: a visibility that is used not to monitor but to magnify and extol. In fact, internet and social media has made possible being seen with great success. Social media celebrity comes exactly from this valence to craft a growing visibility continuously mounting, continuously conquering millions and millions of "likes" and "visualizations". Its best suited aphorism would not be no more: "I see therefore I exist". But "I am seen therefore I exist" (Innerarity, 2004: 132). This is a brave new world: one where skilled media are capable of modulate public attention, either in an active, either in a passive mode. Media are, thus, the great catalyzers of attention and one of the most effective instruments that position, attract and regulate public gaze and collective attention.

And even if they are not the only ones, media are one of the main producers of visibility. They are able to passively and actively modulate visibility because they are incredible powerful dealers of attention. The gathering of attention becomes an incredibly valuable asset and it is precisely this influence of public attention that relates the media to publicness and visibility. Attention becomes also the principle of visibility media deal the most, through those active and passive modulations. Innerarity, for instance, posits attention as the new currency: "Attention has acquired a new meaning as a productive resource and revenue source. The price is not sums of money but relations" (Innerarity, 2004: 135).

Media and communication studies should embrace the exam of this change of the economy to the perceptive and visibility, this immaterial economy of collective attention. The economy of information runs parallel to a most intangible economy: the economy of visibility where mediated attention is governed with some economic principles. In fact, just like in material and economic rationality, success happens by obtaining the maximum of money and rentability, in the immaterial economy of visibility success is only attainable by a maximum of public attention. That's why media (especially social media) are so important to approach visibility as a key topic in communication studies. Media capitalize attention by modulating visibility.

One interesting consequence of this modulation of visibility are the common struggles for visibility who have come to assume such significance today. Even if this is not the time to properly develop these leitmotivs of mediated visibility we should, nevertheless, underscore it as a major means by which political and social fights are carried.

The struggles for visibility indicate how media have become a special visual-symbolic site where different social groups and institutions obtain a notorious presence on publicness that is determinant to the advancement and recognition of his causes. "Mediated visibility is not just a vehicle through which aspects of social and political life are brought to the attention of others: it has become a principal means by which social and political struggles are articulated and carried out. The brutal war of words and images emerging from the war in Iraq illustrates only too well that, in this modern age of mediated visibility, the struggle to be seen and heard, and the struggle 
to make others see and hear, has become an inseparable part of the social and political conflicts of our time" (Thompson, 2004: 49).

Visibility is, thus, a field of production of subjects; and media are the master of ceremonies in the creation of a public awareness based on the maximum attention and full awareness. In other words, media influence social recognition by creating, sustaining and develop visibility relationships. Recognition is a form of social visibility (Brighenti, 2007: 329). Distortions not just in the representation of minority groups (Media Discourse Analysis), but also distortions on the visibility of minority groups (sexual, racial, ethnic minorities) could lead to important misrepresentations. There is verbal, discursive representation. Yet, there is also a visible (more than a visual) imagistic representation.

One of the things communication and media studies should consider is the risk that the successive, elusive and reiterated modulations of visibility fashioned by the media put the field or ecosystem of visibility out of control. This does nor suppose that visibility arrangements cease to exist. It indicates, instead, that these arrangements that constitute different regimes are entering the domain of super-visibility where visibility can become, not a force of development and social presence but a force that paralyzes society. Media representations of immigration is today super-visible just like airplanes crashes have become ubiquitously constant in social imaginary. One can think on the Lamia airplane crash in Colombia, in November 2016, as a good example of that. As images come flooding the collective imaginary, it was world-wide commotion who suddenly rise. A shock but also a shared emotion that froze newspapers - even sports newspapers since the plane carried the Brazilian Chapacoense professional football team. This super-visibility reinforces emotional reactions such tremor and surprise, despair or suffering. Super-visibility can become a risk if it increases the banality of images. Visibilities are everywhere from computer screens, until advertising spot until simulation videos. Super modulations of visibility put in peril our capacity to move and act because we are all paralyzed feeling the pathos involved in every visibility. Images that constantly move us may have the paradoxical effect of freeze us to become passive spectators. In the age of obsessive visibility (Van Weelden, 2005: 8) everything is voraciously consumed. The craving for visibility is in the origin of this super-visibility, that is, an exacerbated modulations media impute to the visible.

The second risk of this situation is that super-visibility banalizes everything. By turning the world visible inside out, by constructing regimes of super-visibility we are transforming banality into a spectacle. Baudrillard talks about hyper-reality and detail this visibility mania as a serious attempt by media to transpose everyday life into a spectacle. "What people really desire is a spectacle of banality. This spectacle of banality is today's true pornography and obscenity. It is the obscene spectacle of nullity (nullité), insignificance and platitude (...). At a time when television and the media in general are less and less capable of accounting for (rendre compte) the worlds's (unbearable) events, they rediscover daily life" (Baudrillard, 2001). So, at a time of fierce media modulation of visibilities banality becomes the most violent piece of information (ex: the exclusive report on the legal divorce between an ex-minister and a television host).

But the greatest risk is, perhaps, the occupation of publicness with banality. The public becomes a judge of everyday life as for example in reality-shows. In the end we have banality squared: banality made visible makes banality a banal activity to watch. So, the super-visibility entails the 
danger to make visibility indecent by continuously exposing it to an endless repetition, a general buildup marking the superiority of quantity of quality. As Baudrillard (2001) notes, there is no more jouissance, enjoyment or pleasure. In other words, super-visibility makes visibility trivial.

When we surrender to visibility, perpetual visibility is what we get. Mediated visibility only exacerbates this structural condition. By introducing distinct modulations, by amplifying visibilities and by intensifying them, media are key elements in the study of visibility.

Communication and media research cannot concentrate themselves exclusively on some effects of media communication. They can and should also consider those effects related to the field of visibility because visibility is a social concept pivoting social action. To attend to communication and media is also to attend to risks, danger and perils mediated visibility carries. Supervisibility and media's visibility modulations may be two phenomena not fully acknowledged, yet they are for sure fully felt in today's world.

\section{Conclusion}

One of the most important things we need to pay attention when discussing visibility is that visibility is beyond the visual. Its symbolic dimension ascribes us to see it as an act of construction of the visible. When the epigraph of Lucretius tells us that we must turn our mind into the visible, he is alerting us to the need of (re)presentate things, to turn our eyes into the visibility. This means, visibility is not a given but a process. And because it is always (re)construction is not just a problem of visual culture but also about media culture and communication enquiry.

In this paper, we tried precisely to approach visibility with a careful eye and we have advanced three reasons why the social category of visibility should be considered a central aspect of communication and media studies.

We have discussed the category of "visibility" by interrogating it through three distinct ways: visibility as a field whose symbolic determination results in the constitution of different regimes of visibility; visibility as a pivot-concept of publicness since it is this public quality that transforms proto-visibility into a full accomplished visibility; and, third, the transmutations and dangers stemmed from media's production of visibility.

In each sub-chapter, we highlighted different ideas related with each principle: so, we need, in the field of visibility, to contemplate inter-visibilities; in public visibilities we need to address proto-visibilities in verge of becoming full-visibilities through the synchrony of collective attention; and in mediated visibility it is imperative to deal with super-visibility as an extreme effect of an intense modulation perpetrated by communication technologies. These three principles constitute three lines of empirical and theoretical investigation: a sociological (symbolic) axis; a collective (publicness) axis; and a technological (media) axis.

Thus, we are suggesting a circuit of visibility between a field and its regimes, a public dimension and its synchronic inter-visibilities and media as fundamental modulators of visibility. Through field, publicness and media we have delineated three angles to see visibility from a communicational standpoint.

The fact of lending visibility to alternative modes of perception has always been a political problem as emphasized by Feminism's struggle to visibility (Van Weelden, 2005: 7). What we 
want suggest is that perception is not just a political problem: it is simultaneously a key topic needing to be considered in its intellectualized form apart from an exclusively sensorial position. Visibility has become a perception problem not just because communication media broke up the traditional frontiers of the visibility but specially because visibility is a communicated, partaken and collective symbolic formulation that have made perception a metaphorical sense to notoriety, recognition and prominence.

As a final note, we would like to synthetize the chapter by stressing how visibilities are constitutive of interactions. The reciprocity of gazes are not just symptoms of the intention people have while interacting (Brighenti, 2010: 24); gazes are also important coordinators of our cognitive and expressive dealing with others. Visibility, just like glances, are interactive forms of communication. How we look, how long and with which aims - both in literal and symbolic dimension - are central elements interfering with the quality (and quantity) of social intercourse. By discussing visibility as a field of reciprocal gazes, inter-visibility and mediated intervention, we wanted to underscore how visibility is also a matter of the communicative encounter with the other.

The way we give others visibility (and possibly invisibility) is crucial to social theory. But social theory cannot be alone and must be accompanied by media and communication theory: because publicness and media are affecting, transforming and alter visibility arrangements, either by its regimes, either by its modulations.

\section{Bibliographical References}

Adorno, T. W. (1954). How to look at television. The Quarterly of Film Radio and Television, Spring, 8 (3): 213-235.

Bataille, G. (1988). The accursed share. An essay on general economy. Vol. I. Consumption. New York, Zone Books.

Baudrillard, J. (2001). Dust Breeding. Ctheory, a095, assessed in December 2016 in www.ctheory. net/articles.aspx $? \mathrm{id}=293$

Benjamin, W. (1999). Experience and Povert. (translation) In M. W. Jennings, H. Eiland \& G. Smith (eds.), Selected Writings: Walter Benjamin, vol 2, 1927-1934. Cambridge MA \& London: The Belknap Press of Harvard University Press.

Boyd, D. \& Marwick, A. (2009). The conundrum of visibility. Journal of Children \& Media, 3 (4): 410-414.

Brighenti, A. M. (2007). Visibility: a category for the social sciences. Current Sociology, 55 (3): 323-342.

Brighenti, A. M. (2008). Visual, visible, ethnographic. Etnografia e Riciera Qualitativa, 1: 2-19.

Brighenti, A. M. (2010). Visibility in social theory and social research. New York, Palgrave Macmilan.

Champagne, P. (1993). La vision médiatique. In P. Bourdieu (ed.), La misère du monde (pp. 61-79). Paris: Seuil.

Debray, R. (1992). Vie et mort de l'image, une histoire du regard en Occident. Paris, Gallimard. 
Dewey, J. (1927). The public and its problems. Athens: Swallow Press, 1991.

Elias, N. (1978). What is sociology?. London: Hutchinson.

Fraser, N. (1992). Rethinking the public sphere: a contribution to the critique of actually existing democracy. In C. Calhoun (ed.), Habermas and the public sphere (pp. 109-142). Cambridge, MA: MIT Press.

Habermas, J. (1991). The structural transformation of the public sphere. Cambridge, Massachusetts: MIT Press.

Innerarity, D. (2004). La ociedad invisible. Madrid: Espasa Calpe.

Kramer, L. (1992). Habermas, history, and critical theory. In C. Calhoun (ed.), Habermas and the public sphere (pp. 236-258). Cambridge, MA: MIT Press.

Mateus, S. (2014). Visibility regimes in mediatized publicness. Matrizes, 8 (2): 259-281.

Mateus, S. (2011). The public as social experience. Comunicação e Sociedade, 19: 275-286.

Mathiesen, T. (1997). The viewer society - Michel Foucault's panopticon revisited. Theoretical Criminology, 1 (2): 215-234.

Negt, O. \& Kluge, A. [1972] (1993). Public sphere and experience: toward an analysis of the bourgeois and proletarian public sphere. P. Labanyi, J. O. Daniel \& A. Oksiloff (trans.). Minneapolis, MN: University of Minnesota Press.

Sennett, R. (1992). The conscience of the eye. The design and social life of cities. New York and London: Norton \& Company.

Simmel, G. (1969). Sociology of the senses: visual interaction. Adapted English trans. In R. E. Park \& E. W. Burgess, Introduction to the science of sociology (pp. 277-294). Chicago: University of Chicago Press.

Tarde, G. (1901). The opinion and the crowd. In Clark TN (ed.), Gabriel Tarde: on communication and social influence: selected.

Thibaut, J.-P. (2001). Frames of visibility in public places. Places, 14 (1): 42-47.

Thompson, J. B. (2005). The new visibility. Theory, Culture and Society, 22 (6): 31-51. R. E. Park \& E. W. Burgess (trans.). Introduction to the Science of Sociology, 3rd edn. Chicago, IL: University of Chicago Press. (Orig. pub. 1908.)

Virilio, P. (1986). Speed and politics: an essay on dromology. M. Polizzotti (trans.). New York: Columbia University Press.

Van Weelden, W. (2005). Viewing: seeing: looking away. Open (In)Visibility, (8): 6-13.

Van Winkel, C. (2005). The regime of visibility. Open (In)Visibility, (8): 14-20.

Weintraub, J. (1997). The theory and politics of the public/private distinction. In J. Weintraub \& K. Kumar (ed.), Public and private in thought and practice - perspectives on a grand dichotomy (pp. 1-42). Chicago \& London: The University of Chicago Press. 